\title{
The scent of life
}

\section{The exquisite complexity of the sense of smell in animals and humans}

A plume of invisible aromas rising from wet grass after a rain shower, the smell of a freshly baked cake, or the hint of a perfume wafting in the air can all evoke strong feelings and memories. Our lives are apparently dominated by the visual sense, but often smells trigger much deeper emotional responses. In fact, olfactory sensations rule much of the behaviour and ecology of a myriad of animal species, including our own.

Our understanding of the mechanisms behind the detection of odours and how the brain reconstructs these stimuli into a 'smell map' of the world has greatly advanced during the past 20 years. Much of the groundbreaking work was done by Richard Axel at Columbia University (New York, NY, USA) and Linda Buck at the Fred Hutchinson Cancer Research Center in Seattle (WA, USA), who shared the 2004 Nobel Prize in Physiology or Medicine "for their discoveries of odorant receptors and the organization of the olfactory system". Their discoveries provide a detailed picture of how odorants are detected by sensory neurons in the olfactory epithelium of the nose, which, in turn, transmits this information to the olfactory bulb that passes the signal on to the olfactory cortex (Fig 1).

The odorant receptors (ORs) on the surface of sensory neurons show significant species-dependent variability and specificity. The mouse, for example, has approximately 1,200 ORs, while humans make do with less than 400 . The primary input from ORs is used in a combinatorial manner to encode odour identities. That is, each OR binds to several odorants, and each odorant is detected by a specific combination of ORs. The brain then translates this specific 'receptor code' into a distinct smell.

Neurons expressing the same OR link with neurons in a segregated area of the olfactory bulb, where a 'first map' of odour inputs is formed. Axons coming from neurons in the olfactory bulb then link with several partly overlapping clusters of neurons in the olfactory cortex. This results in a sensory map that is radically different from that in the olfactory bulb because the olfactory

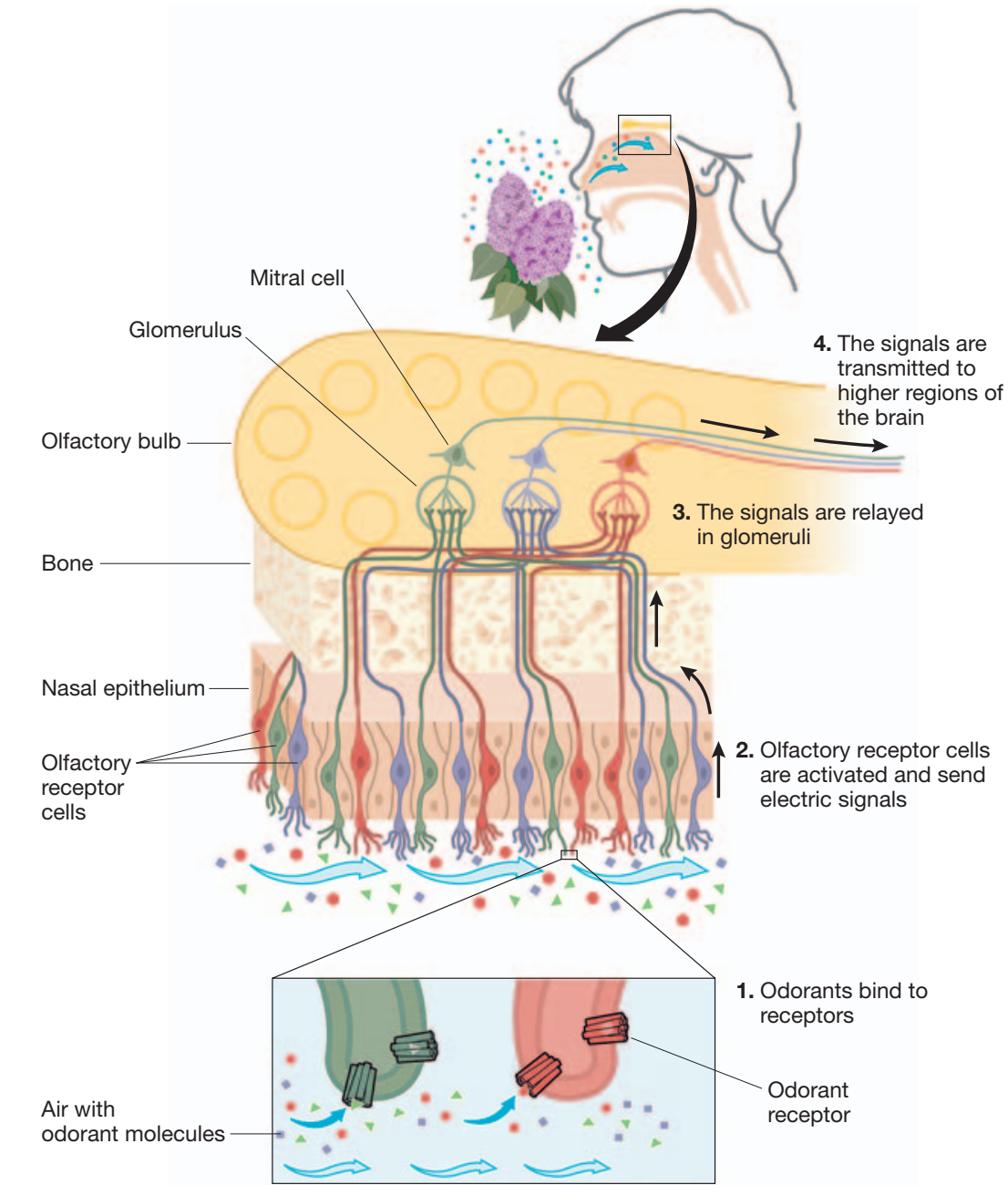

Fig 1 | The human olfactory system. The odorant receptors are localized on olfactory sensory neurons, which occupy a small area in the upper part of the nasal epithelium. Every olfactory receptor cell expresses only one odorant receptor. On activation, signals from olfactory receptor cells are relayed in the glomeruliwell defined micro-regions in the olfactory bulb. Receptor cells of the same type are randomly distributed in the nasal mucosa but converge on the same glomerulus. In the glomerulus, the receptor nerve endings excite mitral cells that forward the signal to higher regions of the brain. Credit: Karolinska Institutet and Nobel Foundation, Stockholm, Sweden.

cortex neurons might receive signals from dozens of different ORs. This "raises the possibility that single cortical neurons can integrate signals from different ORs that detect the same odorant and, thus, perform an initial step in the reconstruction of an odor image from its deconstructed features" (Zou
\& Buck, 2006). Following this scheme, neurons in the olfactory cortex might act as 'coincident detectors' that require input from more than one OR to become active. Supporting this idea about the synthetic capacity of the olfactory cortex, Buck and Zhihua Zou have shown that binary odorant 
mixtures stimulate cortical neurons that were not activated by the initial individual odorants, therefore creating a new odour image in the brain (Zou \& Buck, 2006).

$\mathrm{B}$ ut, further work needs to be carried out to understand how humans and animals in general are able to sense odorants in the first place. "One of the greatest challenges in olfactory science is to correlate ORs with their ligands," commented Bettina Malnic, a molecular neuroscientist at the Universidade de São Paulo in Brazil and Buck's former collaborator. The determination of OR-ligand pairs should reveal how ORs generate diverse perceptions, although these studies are complicated by the extremely large size of the OR family and by the poor functional expression of these receptors in heterologous cells. So far, only a small number of mammalian ORs have been linked to the odorants they recognize.

\section{Our understanding of the mechanisms behind the detection of odours and how the brain reconstructs these stimuli into a 'smell map' of the world has greatly advanced over the past 20 years}

Malnic has attempted to address this problem: "we have recently identified a putative GEF [guanine nucleotide exchange factor], Ric-8B, which is endogenously expressed in olfactory sensory neurons, and which in conjunction with the olfactory G protein Gaolf, promotes OR expression in heterologous cells" (von Dannecker et al, 2006). She believes that the use of the Gaolf-Ric-8B system alone or in combination with accessory proteins increases the chances of identifying OR ligand specificities in heterologous expression systems. "We plan to use this method to explore different aspects of human odorant perception, such as why are some odorants differently perceived by different individuals, or if odorants that elicit similar perceptions or emotions are recognized by overlapping sets of ORs," she said. Her group is also investigating the biological role of Ric-8B in odorant signal transduction, in particular whether this GEF is involved in the apparently paradoxical sensitivity of the olfactory system to tiny concentrations of odorants in vivo, despite the fact that ORs are low-affinity receptors. So far, results show that Ric- $8 \mathrm{~B}$ acts on Gaolf to amplify signal transduction, which is consistent with the role of a GEF.

f puzzling questions and unsolved problems make a research field exciting, then olfactory science is such a field. Scientists still cannot explain how 350-400 smell receptors are able to account for the human ability to detect thousands of different odours. Linked to this is a poor understanding of structureodour relationships, with the subsequent inability to predict the odour properties of a compound from its molecular structure. The dominant theory of smellknown as odotope theory or weak-shape theory-says that the shape of a molecule is recognized by a specific receptor, which therefore determines how it smells. Instead of using a rigid lock-andkey mechanism, the receptors only bind to a small part of an odorant so that many different molecules can activate the same receptor. However, despite its leading status, the shape theory fails to explain why, for example, different molecules can have similar odours and why similar molecules can have dissimilar odours (Sell, 2006).

Among various alternative possibilities that have been proposed, the most controversial is the so-called 'vibration theory of olfaction'. First introduced in the 1930s, it states that the smell of a molecule is determined by intramolecular vibrations rather than by the shape of the molecule. In 1996, Luca Turin, a biophysicist who was working at University College London, UK, revitalized the theory by suggesting that the transduction of molecular vibrations into receptor activation could be mediated by inelastic electron tunnelling (Turin, 1996). Electrons in the odorant receptors would be triggered to tunnel between different energy states if the vibration frequency of the ligand matched the energy difference of these states, thereby activating the receptor. Different odorants have distinctive vibration patterns and different vibration frequencies would be detected by different receptors, which would explain why similar molecules smell different and different molecules can smell the same.

Scientists still cannot explain how 350-400 smell receptors are able to account for the human ability to detect thousands of different odours
Turin's idea did not receive much attention until 2003, after science writer Chandler Burr wrote a book about Turin, his 'heretical' theory and how the established scientific community purportedly ignored it (Burr, 2003). Suddenly, the discussion about the vibration theory of smell was immersed in a harsh controversy about the correctness of scientific practice (Solomon, 2006). But can vibration theory help to explain odour perception?

Researchers such as Leslie Vosshall, of the Rockefeller University in New York (NY, USA), have provided some evidence that molecular vibrations alone cannot explain the perceived smell of an odorous molecule. Together with Andreas Keller, Vosshall asked volunteers to smell three sets of substances for which Turin had previously indicated the behaviour according to the vibration theory, and then recorded their olfactory sensations (Keller \& Vosshall, 2004). In each case, the tests failed to fulfil Turin's prediction. "We didn't disprove the vibration theory. We just didn't find anything to support it," Vosshall commented in a press release. "All our data are consistent with the shape theory, but don't prove the shape theory" (Rockefeller University, 2004).

$\mathrm{H}$ owever, a group from University College London, UK, led by Marshall Stoneham, recently analysed Turin's theory and found that it is consistent with the underlying physics and with known features of smell (Brookes et al, 2007). "The full significance of Luca Turin's idea that we investigated in the paper [...] can only be judged properly after more experimental work has been done," said Andrew Horsfield, one of the authors of the study. "However, we can say some things even now." The conventional theory has limited predictive or explanatory power, so some other theory is probably needed. He explained: "At present, the only other game in town is Turin's theory, and so far it looks promising. This is the main finding of our article. In it we cite some examples of its success: it explains why some boranes smell sulphuraceous, why humans can distinguish nickelocene from ferrocene, and why some animals can distinguish deuterated odorants from undeuterated ones. The odotope theory struggles to explain these."

"The [Brookes et al] paper is a major advance," commented Turin. "My original 1996 paper proceeded by analogy with the 
known electron tunneling spectroscopy mechanism in metal-insulator-metal junctions and proposed that something similar may be at work in olfaction. The open question was: how similar? There are no metallic conductors in biological systems, and a proper mechanism based on biological materials was needed." Stoneham and colleagues proposed a 'swipe card' model, which suggests that the vibration spectrum of an odorant molecule is read and picked up by receptors for non-mechanical actuation, but does not exclude the possibility of selective docking based on the molecular shape and size of the odorant.

\section{Stoneham and colleagues}

proposed a 'swipe card' model, which suggests that the vibration spectrum of an odorant molecule is read and picked up by receptors for non-mechanical actuation...

Since 2001, Turin has been working with Flexitral, a private company in Chantilly (VA, USA), which produces odorants for industrial applications based on Turin's theories (Turin, 2002). Flexitral claims that its products are determined after synthesizing less than ten molecules-an extraordinary rate of success when compared to the rest of the flavour and fragrance industry, in which less than one in a thousand molecules make it to the market. Nonetheless, there are those in the perfume technology business who believe that recent progress in elucidating the mechanism of olfaction-with its multilevel receptor-neuronal signal integration process-will not necessarily make their work easier. "It would therefore seem that consistently accurate prediction of odours will not be possible for a very considerable time," wrote flavour chemist Charles Sell, "and not until a great amount of further research has been completed, the cost of which could not be borne by the flavour and fragrance industry" (Sell, 2006).

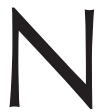
ew surprises continue to emerge as the scientific exploration of the sense of smell proceeds. One example is the recent discovery in Vosshall's laboratory, where they use Drosophila fruit flies as a model to understand how the brain interprets olfactory signals in the environment. As in many insects,

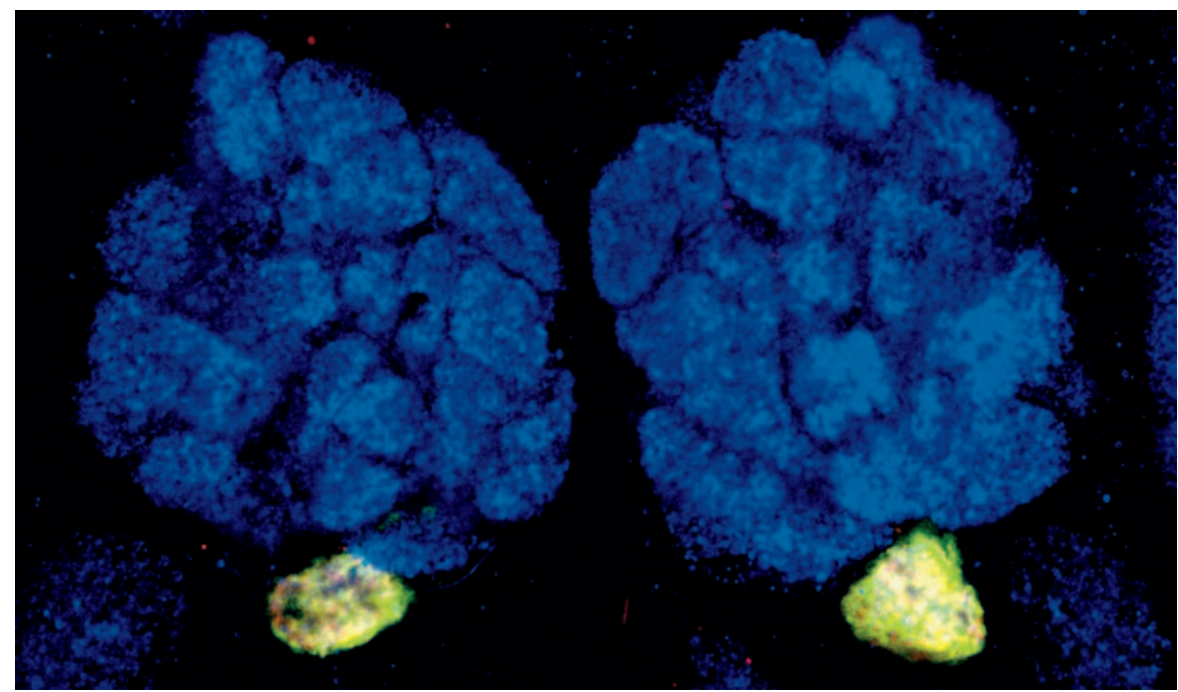

Fig $2 \mid \mathrm{CO}_{2}$-sensitive neurons expressing $\mathrm{Gr} 21 a$ (green) and $\mathrm{Gr} 63 a$ (red), proteins that together are necessary for $\mathrm{CO}_{2}$ detection in Drosophila. The neurons target a specific region of the fly brain, which is dedicated to processing the smell of $\mathrm{CO}_{2}$. Credit: Vosshall Laboratory, Rockefeller University. Reprinted with permission from Macmillian Publishers Ltd [Nature, Jones et al, 2007].

fruit flies can detect minor changes in the concentration of $\mathrm{CO}_{2}$ and other gases with a high degree of sensitivity-for example, mosquitoes follow $\mathrm{CO}_{2}$-trails to locate potential hosts. However, until Vosshall's recent findings it was not clear how insects are able to smell $\mathrm{CO}_{2}$ and other small volatiles. "The main finding of this work is that $\mathrm{CO}_{2}$ chemoreception in insects depends critically on two membrane proteins, Gr21a and Gr63a," Vosshall said (Jones et al, 2007; Fig 2). "We were excited by this because it represents a very different mechanism of gas sensation than has previously been documented." For example, Vosshall explained, nitric oxide and oxygen are sensed by cytoplasmic haemcontaining proteins, not membrane proteins. As the gustatory receptor (Gr) genes are members of the same ancestral family as the insect odorant receptors, it suggests that insects have invented new ways to detect small molecules, including $\mathrm{CO}_{2}$. "We think this work may have applications in public health," remarked Vosshall. "Because mosquitoes pay attention to both body odour and exhaled breath, it is conceivable that small-molecule inhibitors of the insect $\mathrm{CO}_{2}$ receptor could be a new tool to make more effective insect repellents." Yet, it is still unclear whether these receptors actually bind to gaseous $\mathrm{CO}_{2}$ or to its commonly occurring metabolite bicarbonate, or whether they respond to the elevation of $\mathrm{pH}$ induced by $\mathrm{CO}_{2}$, or if another mechanism plays a role.

\section{Fish [...] use a complicated network of chemical stimuli that pervade water-based ecosystems}

Many other animals also rely on sophisticated chemosensorial communication to guide their reproductive and feeding behaviour. Fish, for example, use a complicated network of chemical stimuli that pervade water-based ecosystems. Several studies have focused on the sea lamprey, an evolutionarily ancient eel-like predator that feeds on other fish. Sea lampreys suck the body fluids out of their prey, often leaving them dying or dead (Fig 3). The life cycle of the sea lamprey consists of two stages: a larval phase spent filter-feeding, burrowed in the bottom sediments of freshwater streams for up to 20 years, and a short parasitic phase during which the adult lampreys move to the open sea or lakes to feed on other fish. After one year, the adults interrupt their parasitic behaviour, stop eating and return to streams to spawn-although not necessarily the same ones where they hatched.

It has been known for some time that a chemoattractant released by stream-dwelling 

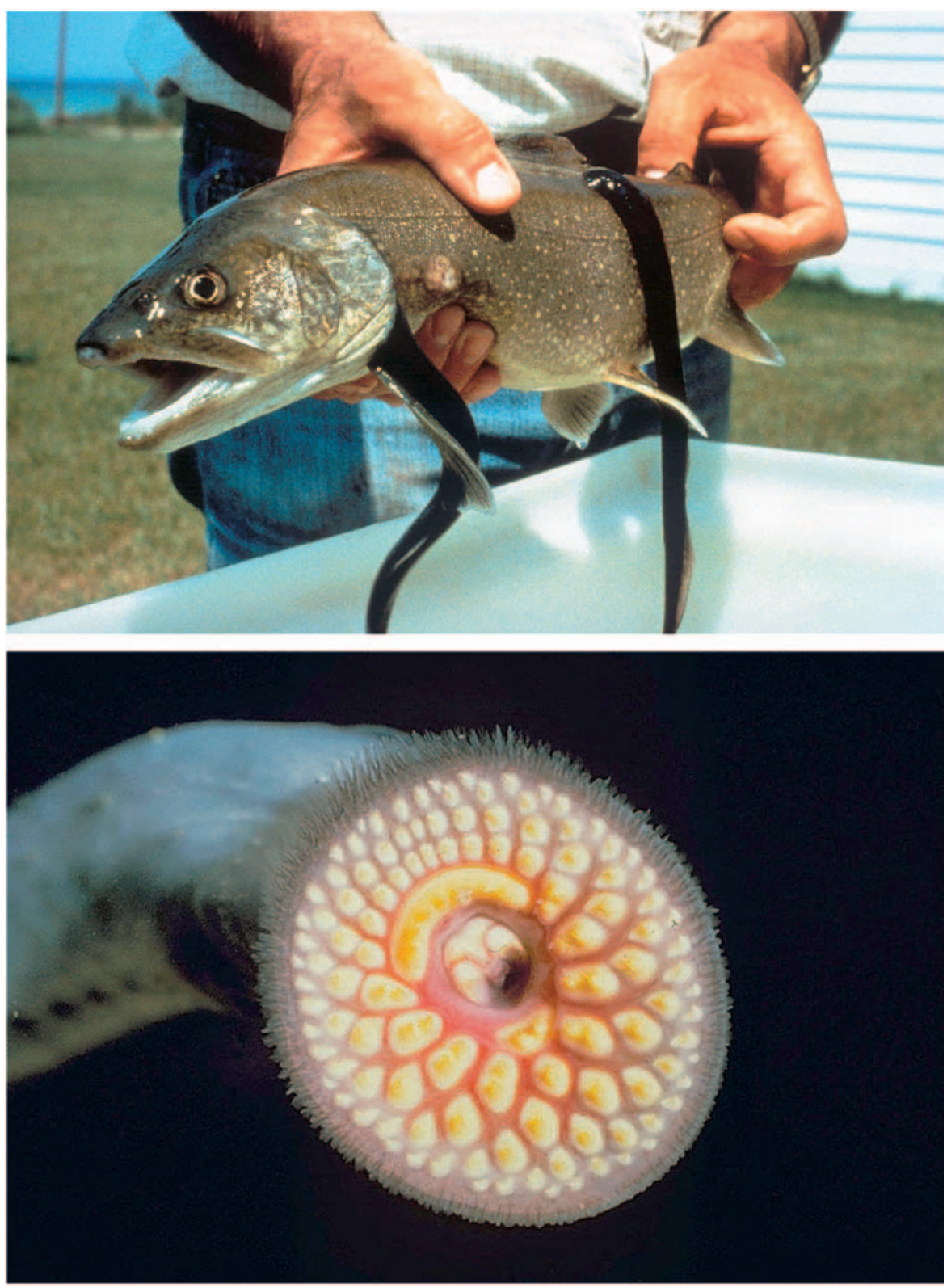

Fig 3 Sea Lamprey (Petromyzon marinus) on lake trout (top) and detail of the oral disc (bottom). Sea lampreys are found in the Great Lakes region and injure lake trout and many other fishes, from which they suck out the body fluids by using teeth and a grasping tongue. Credit: US Fish and Wildlife Service.

lamprey larvae drives migratory adults to suitable spawning and nursery habitats-and that this odorous trail is deployed with extraordinary potency. A single larva, which weighs about $1 \mathrm{~g}$, can activate around $400 \mathrm{l}$ of water per hour. A group led by Peter Sorensen at the University of Minnesota in St Paul (MN, USA), finally disclosed the exact nature of what can be considered "the first migratory pheromone identified in a verte-

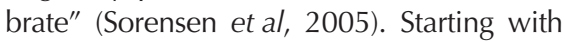

8,000 I of water that had contained 35,000 larvae, Sorensen and colleagues isolated a mixture of three compounds-petromyzosterol disulphate, petromyzonol sulphate and
The field of olfactory science is undoubtedly exciting and now offering many more practical applications than one might imagine

petromyzonamine disulphate(PADS)—which constitute the lamprey pheromone, with PADS as the crucial component (Sorensen et al, 2005). The three substances are all sulphate steroids and therefore chemically, and possibly biosynthetically, related.

Interestingly, PADS closely resembles squalamine (see sidebar), the first in a series of aminosterols with antimicrobial activity that were isolated from the dogfish shark Squalus acanthias (Moore et al, 1993; Rao et al, 2000). Squalamine also shows antiangiogenic and antitumour properties, and is now in clinical development for the treatment of solid tumours in conjunction with chemotherapy. "How marvelous, yet sensible, that the pathways which produce aminosterols should be conserved across evolutionary time," Sorensen commented. "Of course, in retrospect, it makes perfect sense that potent antiangiogenic and antimicrobial agents such as squalamine should be produced in such great quantities that fishes have evolved the ability to detect compounds leeching from conspecifics and to use them as indicators of each other's presence, i.e. as pheromones."

According to Sorensen, the question is how many of the more than 26,000 fish species use aminosterols in similar ways, and how specialized and diverse might be the pathways for their production. Besides adding to the knowledge of fish chemical ecology, understanding the sense of smell of the sea lamprey has obvious potential applications. The species invaded the Great Lakes in North America 100 years ago and is causing severe damage among fish stocks with associated financial losses. "Petromyzonamine disulfate is now being developed as a pheromonal attractant to help control the sea lamprey problem in the Great Lakes, the first application of a vertebrate pheromone to invasive species control," Sorensen said.

"Although we do not know what squalamine's function in the dogfish shark is, we do know that the aminosterol is a potent broad-spectrum antimicrobial agent," said Michael Zasloff from Georgetown University in Washington DC, USA, who discovered the molecule. "We also know that the larval lamprey live on microbes as their sole food source. I assume that squalamine provides some sort of protection," he added. "The secretion of PADS from developing larvae would signal to the adults searching for a spawning site that these larvae were healthy: antimicrobial defenses 


\section{A BIOCHEMICAL TALE}

Squalamine

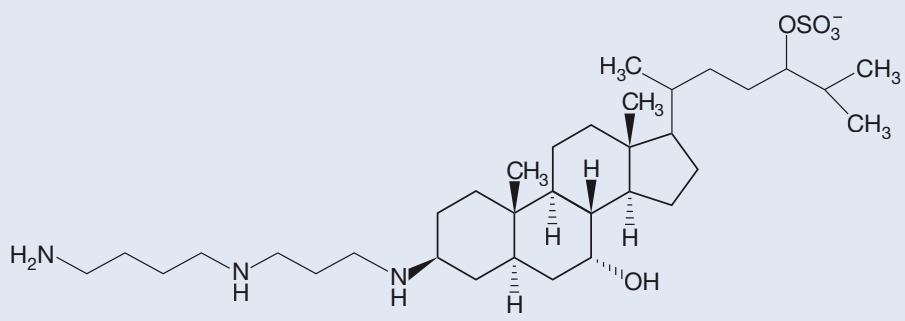

PADS (petromyzonamine disulphate)<smiles>CC(C)C(CCC(C)C1CC[C@@H]2[C@]1(C)CC[C@@H]1[C@@]3(C)CC[C@@H](NCCCN4CCCC4=O)C[C@@H]3C[C@@H](OS(=O)(=O)[O-])[C@]12C)OS(=O)(=O)[O-]</smiles>

MSI-1436

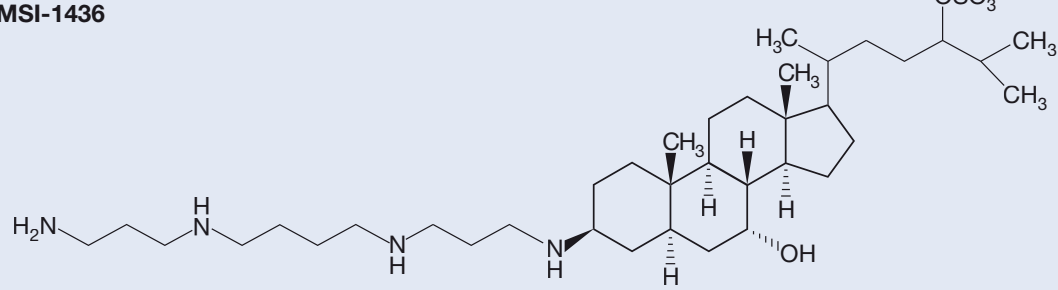

Squalamine is more than just a pheromone. "There is an aspect of the biology of the lamprey pheromone that is rather perplexing," Zasloff said. "After the larvae undergo metamorphosis and venture into lake waters, they begin to feed and grow larger. At a certain point in their maturation they stop eating, become sexually competent and ready to begin spawning, and become receptive to PADS." During this PADS-driven period, when they migrate back to the stream, they do not eat, depending solely on stored triglycerides. "How can this happen? Well, another aminosterol we isolated from the dogfish sharkMSI-1436 - might help explain this part of the PADS-story," Zasloff proposed. MSI-1436 is identical to squalamine, apart for the presence of a spermine rather than a spermidine (Rao et al, 2000). This molecule apparently acts on the hypothalamus, and causes rats and mice to stop eating and consume stored adipose tissue. Unlike what occurs in simple anorexia, MSI-1436-induced weight loss is not associated with diminished physical activity and animals maintain a normal metabolic rate. Furthermore, MSI-1436 mobilizes stored triglycerides from the liver in mice that have developed fatty livers, under conditions in which simple food restriction cannot. In the dogfish shark, MSI-1436 might be responsible for the sporadic feeding behaviour of the animal, which normally eats once every two weeks.

"So here's the thought. Maybe, the adult lamprey undergoes a phase of sexual maturation when it reaches a certain weight and has stored the necessary amount of energy, and can recognize the PADS pheromone," said Zasloff. At the same time it might also synthesize MSI-1436, he believes. When the animal recognizes PADS, it simultaneously loses its desire to eat, begins to consume stored energy and returns homeward to mate without distraction. "This is a fascinating story," concluded Zasloff. "The responsiveness of mammals to these compounds suggests that receptors exist in mammals, and begs the question as to whether or not they also produce squalamine-like compounds." So far, no such compounds have been described, although no extensive search has been conducted. As Zasloff pointed out, by virtue of their physical properties - they are water-soluble amphipathic steroids-they would not have been discovered anyway, just as they had been missed in the extensive studies conducted over the years in the search for steroids and bile alcohols in Squalus acanthias. were robust, signifying both an excellent food source and the presence of an environment supporting the development of healthy larvae."

The field of olfactory science is undoubtedly exciting and now offering many more practical applications than one might imagine. However, unfolding the mysteries of smells and the way we perceive them requires a great deal more time and effort. But like the hunting hound following the scent of its quarry, researchers seem to be on the right track.

\section{REFERENCES}

Brookes JC, Hartoutsiou F, Horsfield AP,

Stoneham AM (2007) Could humans recognize odor by phonon assisted tunnelling? Phys Rev Lett 98: doi:10.1103/PhysRevLett.98.038101

Burr C (2003) The Emperor of Scent: A Story of Perfume, Obsession, and the Last Mystery of the Senses. New York, NY, USA: Random House Jones WD, Cayirlioglu P, Kadow IG, Vosshall LB (2007) Two chemosensory receptors together mediate carbon dioxide detection in Drosophila. Nature 445: 86-90

Keller A, Vosshall LB (2004) A psychophysical test of the vibration theory of olfaction. Nat Neurosci 7:337-338

Moore KS, Wehrli S, Roder H, Rogers M, Forrest Jr JN, McCrimmon D, Zasloff M (1993) Squalamine: an aminosterol antibiotic from the shark. Proc Natl Acad Sci USA 90: 1354-1358

Rao MN et al (2000) Aminosterols from the dogfish shark Squalus acanthias. I Nat Prod 63: 631-635

Rockefeller University (2004) Rockefeller University scientists take on controversial and widely publicized 'vibration theory' of smell. New York, NY, USA: Rockefeller University http://runews.rockefeller.edu

Sell C (2006) On the unpredictability of odor. Angew Chem Int Ed 45: 6254-6261

Solomon M (2006) On smell and scientific practice. Science 313: 763-764

Sorensen PW, Fine JM, Dvornikovs V, Jeffrey CS, Shao F, Wang J, Vrieze LA, Anderson KR, Hoye TR (2005) Mixture of new sulfated steroids functions as a migratory pheromone in the sea lamprey. Nat Chem Biol 1: 324-328

Turin L (1996) A spectroscopic mechanism for primary olfactory perception. Chem Senses $\mathbf{2 1}$ : 773-791

Turin L (2002) A method for the calculation of odor character from molecular structure. / Theor Biol 216: 367-385

von Dannecker LEC, Mercadante AF, Malnic B (2006) Ric-8B promotes functional expression of odorant receptors. Proc Natl Acad Sci USA 103: 9310-9314

Zou Z, Buck LB (2006) Combinatorial effects of odorant mixes in olfactory cortex. Science $\mathbf{3 1 1}$ : 1477-1481

\section{Andrea Rinaldi}

doi:10.1038/sj.embor.7401029 